\title{
Semi-quantitative indices of cardiac uptake in patients with suspected cardiac amyloidosis undergoing 99mTc-HMDP scintigraphy
}

\author{
Chiara Gallini, $\mathrm{MD}, \mathrm{PhD},{ }^{\mathrm{a}}$ Francesca Tutino, $\mathrm{MD},{ }^{\mathrm{a}}$ Raffaele Martone, $\mathrm{MD},{ }^{\mathrm{b}}$

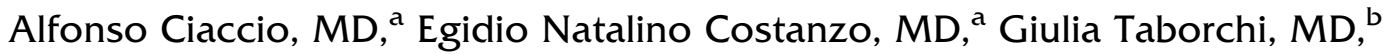 \\ Sofia Morini, MD, ${ }^{\mathrm{b}}$ Simone Bartolini, MD, ${ }^{\mathrm{b}}$ Silvia Farsetti, MD, ${ }^{\mathrm{d}}$ \\ Carlo Di Mario, MD, PhD, ${ }^{\mathrm{c}}$ Federico Perfetto, MD, PhD, ${ }^{\mathrm{d}}$ and Francesco Cappelli, \\ $M D^{\text {d,e }}$ \\ a Nuclear Medicine Unit, Careggi University Hospital, Florence, Italy \\ b Cardiovascular and Thoracic Department, Careggi University Hospital, Florence, Italy \\ c Interventional Structural Cardiology Division, Department of Heart, Lung and Vessels, Careggi \\ University Hospital, Florence, Italy \\ d Tuscan Regional Amyloid Centre, Careggi University Hospital, Florence, Italy \\ e Intensive Cardiac Care Unit, Interventional Structural Cardiology Division, Department of Heart, \\ Lung and Vessels, Careggi University Hospital, Florence, Italy
}

Received Oct 9, 2018; accepted Jan 11, 2019

doi:10.1007/s12350-019-01643-w

Background. 99mTc-HMDP scintigraphy has proved its efficacy in non-invasive diagnosis of cardiac amyloidosis (CA) and is currently interpreted according to the Perugini qualitative assessment. Several semi-quantitative indices have been proposed to overcome inherent possible limitations of visual grading. Our aim was to comparatively evaluate six different indices and their diagnostic performance.

Methods. We retrospectively reviewed scintigraphy of 76 patients (53 ATTR, 12 AL, 11 LVH) who underwent diagnostic evaluation at our centre. ROC-curve analysis was performed to identify optimal cut-off and relative diagnostic accuracy of six different indices (of which one was proposed for the first time), both in identifying $\mathrm{CA}$ patients and in discriminating patients according to their Perugini score.

Results. Heart/Whole-body ratios proved to be the most accurate (100\%) in identifying CA patients. Heart/Pelvis ratio (with soft tissue background correction) offered acceptable accuracy $(98 \%)$, with the largest area under the curve (AUC) $(0.98)$ in discriminating patients with Perugini $\geq 2$. Heart/Contralateral Lung ratio confirmed to be exposed to confounding background noise in case of simultaneous lung uptake. Heart/Skull ratio had the worst performance, with six false-negative patients in ATTR identification.

Conclusion. Heart/Whole-body ratios may be robust and effective semi-quantitative indices for the evaluation of CA by means of scintigraphy (J Nucl Cardiol 2019)

Key Words: Amyloid heart disease $\cdot$ SPECT $\cdot$ molecular imaging agents

Electronic supplementary material The online version of this article (https://doi.org/10.1007/s12350-019-01643-w) contains supplementary material, which is available to authorized users.

The authors of this article have provided a PowerPoint file, available for download at SpringerLink, which summarises the contents of the paper and is free for re-use at meetings and presentations. Search for the article DOI on SpringerLink.com.
Reprint requests: Francesco Cappelli, MD, Intensive Cardiac Care Unit, Interventional Structural Cardiology Division, Department of Heart, Lung and Vessels, Careggi University Hospital, Largo Brambilla 3, 50134, Florence, Italy; cappellifrancesco@inwind.it $1071-3581 / \$ 34.00$

Copyright (c) 2019 American Society of Nuclear Cardiology. 


\begin{tabular}{|ll|}
\hline Abbreviation & \\
$\mathrm{H} / \mathrm{WBr}$ & Heart/whole-body rectangular \\
& ROI ratio \\
$\mathrm{H} / \mathrm{WBp}$ & Heart/whole-body profile ROI ratio \\
$\mathrm{H} / \mathrm{P}$ & Heart/Pelvis Index \\
$\mathrm{H} / \mathrm{S}$ & Heart/Skull Index \\
$\mathrm{H} / \mathrm{P}-\mathrm{bkg}$ & Heart/pelvis ratio with tissue \\
& background correction \\
$\mathrm{H} / \mathrm{CL}$ & Heart/contralateral lung ratio \\
99mTc- & 99mTc-Hydroxymethylene \\
HMDP & diphosphonate \\
99mTc- & 99mTc-3,3-diphosphono- \\
DPD & 1,2-propanodicarboxylic acid \\
99mTc- & $99 \mathrm{mTc}-$ Pyrophosphate \\
PYP & \\
\hline
\end{tabular}

Systemic amyloidosis is characterized by extracellular deposition of protein-derived fibrils in different tissues and organs, including the heart. ${ }^{1}$ Two types of amyloid commonly infiltrate the heart: immunoglobulin light-chain (AL) amyloid and transthyretin (TTR) amyloid.

In recent years, total body scintigraphy with diphosphonates, either with 99mTc-3,3-diphosphono1,2-propanodicarboxylic acid (99mTc-DPD), 99mTcHydroxymethylene diphosphonate (99mTc-HMDP) and 99mTc-Pyrophosphate (99mTc-PYP) has gained an important role in diagnosis of cardiac amyloidosis and in defining the TTR subtype. ${ }^{2-12}$ Since the work by Perugini et al., a visual score based on the strength of cardiac uptake compared to bone uptake has become of routine use to describe patients that undergo total body scintigraphy in the suspect of cardiac amyloidosis. Several semi-quantitative indices have been proposed to describe cardiac uptake of $99 \mathrm{mTc}$-diphosphonates in cardiac amyloidosis, without agreement or clear demonstration of superiority of one over another. ${ }^{6,9-11}$ Several reports, mainly based on planar scintigraphy, evaluated the clinical use of a ratio between heart tracer retention and whole-body retention (H/WB ratio), ${ }^{11,12}$ while other authors tested the accuracy of a ratio between heart retention and skull retention (H/S ratio). ${ }^{9,10}$ On the other hand, in the United States, following the work by Bokhary et al., the most commonly used method is the heart-to-contralateral lung ratio $(\mathrm{H} /$ $\mathrm{CL}$ ), obtained by drawing a region of interest (ROI) over the heart corrected for a contralateral ROI counts. ${ }^{6,13}$

To the best of our knowledge, no comprehensive clinical comparison of different semi-quantitative indices in amyloid identification has been till now performed.

The purpose of our study is to compare, in a population of patients that underwent 99mTc-HMDP total body scintigraphy during multidisciplinary evaluation for suspected cardiac amyloidosis, already tested semiquantitative indices of cardiac uptake and to evaluate their reliability and handling in routine use. Moreover, we evaluated a new semi-quantitative index based on the ratio between heart retention and hip retention, comparing its diagnostic performance to the already published ones.

Furthermore, we aim to test if different semiquantitative indices were able to discriminate between Perugini 0-1 and Perugini 2-3 visual score, a distinction that reached a great clinical impact since the validation of Gillmore diagnostic algorithm. ${ }^{14}$

\section{MATERIALS AND METHODS}

From the Tuscan Regional Amyloidosis Centre database, we retrospectively evaluated all patients that underwent 99mTc-HMDP total body scintigraphy as a part of the usual diagnostic workup to exclude or confirm a suspected cardiac amyloidosis. All patients underwent physical examination, electrocardiogram (ECG), transthoracic echocardiography and blood tests including NT-proBNP, serum and urinary immunofixation and serum-free light chain measurement. In case of inconclusive results patients underwent cardiac MRI.

All patients gave written informed consent for their clinical records to be used for research purposes, in accordance with Institutional Review Board guidelines.

\section{Diagnosis of Amyloidosis and Cardiac Involvement Definition}

Diagnosis of light-chain (AL) amyloidosis was made by biopsy of abdominal fat pad or biopsy of an involved organ, which demonstrated the typical Congo Red birefringence when viewed under polarized light. All positive biopsies demonstrated typical Congo Red birefringence under polarized light and staining by anti-k or anti- $\lambda$ light-chain antibodies, combined with elevated serum or urine levels of the corresponding monoclonal light chain.

Diagnosis of TTR amyloidosis was made invasively by staining of tissue biopsy with anti-TTR antibodies (irrespectively of cardiac uptake at bone-tracer scintigraphy), or according to the non-invasive algorithm suggested by Gillmore and colleagues ${ }^{14}$ whenever a positive tissue biopsy was not available: that required a combination of a Perugini grade 2 or 3 bone-tracer cardiac uptake, together with the absence of a detectable serum/urinary monoclonal protein, in a patient with echocardiogram and/or MRI suggestive of cardiac amyloidosis. 
Diagnosis of mutated (ATTRm) or wild-type (ATTRwt) amyloidosis was made according to presence or absence of mutation at genotyping.

Diagnosis of cardiac involvement in systemic amyloidosis was made according to the International Society of Amyloidosis criteria. $^{15}$

Patients were considered to have left ventricular hypertrophy $(\mathrm{LVH})$ not related to amyloid infiltration if they had a negative cardiac biopsy or at least two negative non-cardiac biopsies with no suggestive signs of cardiac amyloidosis at non-invasive imaging evaluation (99mTc-HMDP total body scintigraphy, echocardiography and MRI), in the absence of amyloidogenic mutation at genotyping.

\section{Standard and Tissue Doppler Imaging Echocardiography}

Patients were referred to our laboratory for echocardiographic study. Echocardiography was performed using a Vivid 9 System (Vingmed, General Electric, Horten, Norway) equipped with a $3 \mathrm{~S}$ probe. According to the standards of the American Society of Echocardiography, ${ }^{16}$ the following parameters were assessed: end-diastolic thickness of interventricular septum (IVS) and LV posterior wall (PW), LV enddiastolic and end-systolic volumes (LVEDV and LVESV, respectively), ejection fraction (estimated with the biplane Simpson method), and tricuspid annular plane systolic excursion (TAPSE). We also evaluated pulsed tissue Doppler imaging-derived early diastolic peak velocity of mitral annulus (E'), to perform the $\mathrm{E} / \mathrm{E}$ ' ratio as an index of $\mathrm{LV}$ filling pressure.

\section{9mTc-HMDP Scintigraphy}

All patients had 99mTc-HMDP whole-body scintigraphy (HMDP-TB). 99mTc-HMDP was prepared from the commercial kit $\left(\right.$ OSTEOCIS ${ }^{\circledR}$ ) following the prescriptions of the manufacturer. All the preparations were tested for total hydrolysed and free $99 \mathrm{mTc}(<5 \%)$ and for radiochemical purity $(>95 \%)$. Each patient received intravenous 700$740 \mathrm{MBq}$ of $99 \mathrm{mTc}-\mathrm{HMDP}$ and a whole-body scan (anterior and posterior projections) was performed 150 minutes later in a $256 * 1024$ matrix. Images were acquired in two different gamma cameras, Discovery (GE) and Ecam (Siemens), using low energy, high-resolution collimators and an appropriate scan speed to reach over 2.000.000 count. Each scintigraphy was evaluated by Perugini visual score as previously published. $^{2}$

\section{Semi-quantitative Analysis}

In all patients, 99mTc-HMDP cardiac uptake was assessed using the following semi-quantitative simplified indices: Heart/whole-body rectangular ROI ratio (H/WBr); Heart/whole-body profile ROI ratio (H/WBp); Heart/Pelvis Index $(\mathrm{H} / \mathrm{P})$, Heart/pelvis ratio with tissue background correction (H/P-bkg), Heart/Skull Index (H/S), and Heart/ Contralateral Lung ratio (H/CL) (Figure 1).

- Heart/Whole-Body rectangular ROI ratio (H/ WBr): the following ROI were drawn (anterior projection): a rectangular one embracing the wholebody profile; three anatomical ROI traced along the bladder and the two kidneys, and a heart ROI. Tracer uptake was quantified as total counts for each ROI. The semi-quantitative index was calculated as follows:

$$
\frac{\text { heart ROI }}{\text { WBrect. ROI }-(\text { bladder ROI + kidneys ROIs })}
$$

- Heart/Whole-Body profile ROI ratio (H/WBp): it is analogous to the previous index, with the exception that WB ROI is drawn along the anatomical body profile, rather than along a rectangular simplified shape.

- Heart/pelvis ratio (H/P): the following ROI were drawn: (anterior projection) a heart ROI and (posterior projection) an anatomical ROI around the pelvis and the sacrum profile. Tracer uptake was quantified as mean counts for each ROI. The semi-quantitative index was calculated as follows:

$$
\frac{\text { heart ROI }}{\text { pelvis Rol }}
$$

- Heart/pelvis ratio with tissue background correction (H/P-bkg): the following ROI were drawn (anterior projection): a simplified circular ROI around the heart and a specular contralateral ROI in the right hemithorax (bkg1); (posterior projection) a simplified ovalar ROI around a sacroiliac joint; a simplified little rectangular ROI over the abdomen (bkg2). Tracer uptake was quantified as mean counts for each ROI. The semi-quantitative index was calculated as follows:

$$
\frac{\text { heart ROI }-b k g 1}{\text { Sacroiliac joint ROI }-b k g 2}
$$

- Heart/skull ratio (H/S): the following ROI were drawn: two heart ROI (anterior and posterior projection) and two simplified rectangular ROI around the skull (anterior and posterior projection). Tracer uptake was quantified as mean counts for each ROI. The semi-quantitative index was calculated as follows:

$$
\frac{\text { arithmetic mean heart ROIs }}{\text { arithmeteic mean skull ROIs }}
$$

- Heart/contralateral lung (H/CL): the following ROI were drawn: a simplified circular ROI around the 

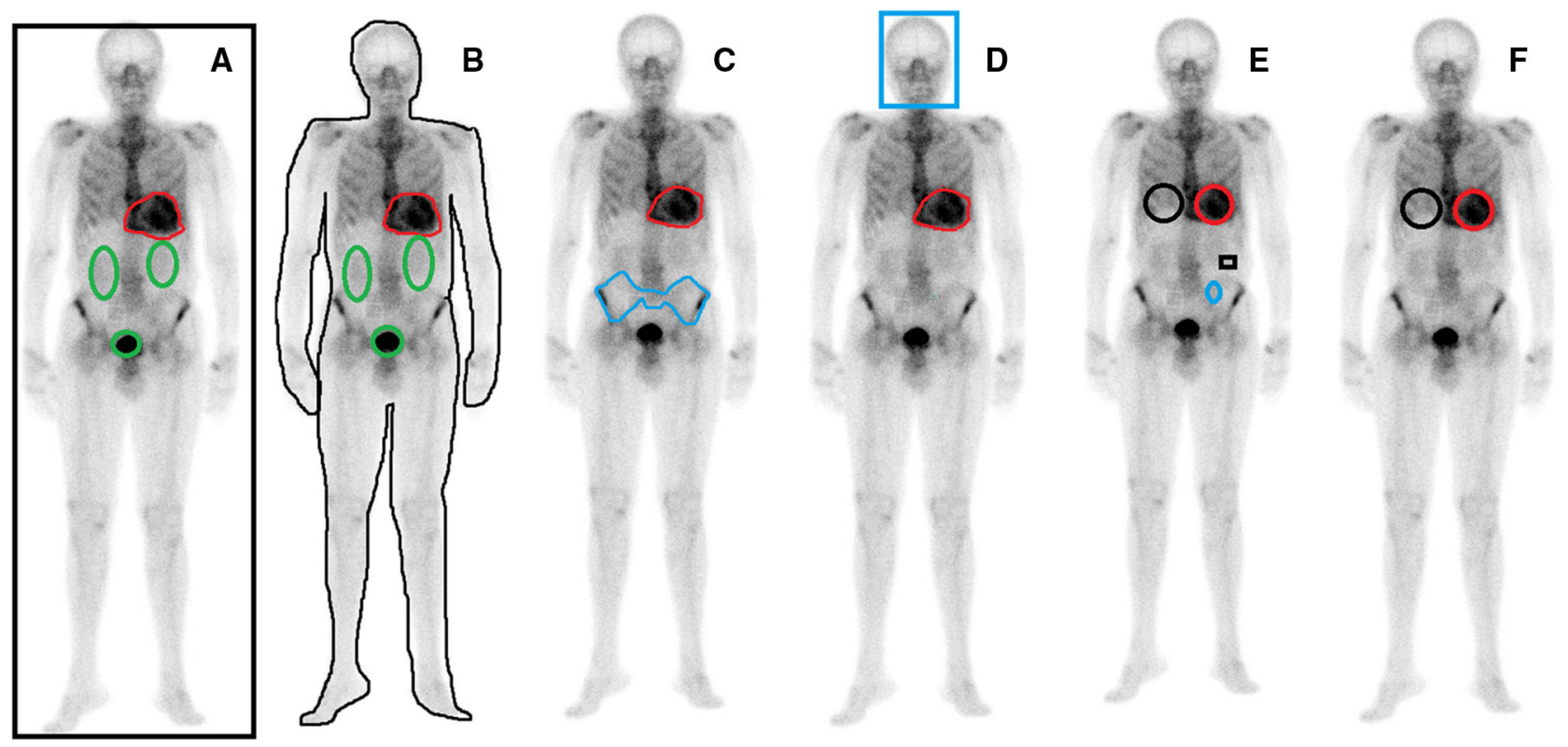

Figure 1. Representation of ROIs' drawing method for index calculation. A Heart/whole-body rectangular ROI ratio (H/WBr); B Heart/Whole-Body profile ROI ratio (H/WBp); C Heart/Pelvis Index (H/P); D Heart/Skull Index (H/S); E Heart/pelvis ratio with tissue background correction (H/ P-bkg); F Heart/contralateral lung ratio (H/CL).

heart and a specular contralateral ROI in the right hemithorax (contralateral lung; CL) (anterior projection). Tracer uptake was quantified as mean counts for each ROI. The semi-quantitative index was calculated as follows:

$$
\frac{\text { heart ROI }}{\text { CLROI }}
$$

\section{STATISTICAL ANALYSIS}

Continuous variables are expressed as mean values and standard deviation, and categorical variables as frequencies and percentages. Multiple comparisons for continuous variables were performed with ANOVA followed by Scheffè test for post hoc analyses.

For each heart-retention semi-quantitative index, sensitivity and specificity were defined by ROC curves analysis, grouping the patients into two categories: ATTR, as positive and AL and non-amyloidosis, as negative. Moreover, we defined for each semi-quantitative index, by ROC curve analysis, the best cut-off value able to discriminate Perugini $0-1$ visual score from Perugini 2-3 visual score.

All statistical analyses and comparisons were performed with SPSS 20 Version IBM Package and we considered statistical significance a $P$ value $<0.05$.

All statistical analyses and comparisons were performed with SPSS 20 Version IBM Package and we considered statistical significance a $P$ value $<0.05$.
To check for consistency of measures obtained by semi-quantitative scoring, we performed a formal analysis of reproducibility (inter-observer variability) and repeatability (intra-observer variability) on a randomly selected sample of 20 patients; results were compered by mean of Intraclass correlation coefficient (ICC) with a cut-off of 0.90 .

\section{RESULTS}

From Tuscan Regional Amyloid Center database retrospective analysis, we identified 76 subjects that underwent 99mTc-HMDP scintigraphy during evaluation for suspected cardiac amyloidosis. At the end of multidisciplinary assessment, 12 patients were affected by AL cardiac amyloidosis, 53 patients were affected by TTR cardiac amyloidosis (16 ATTRm, 37 ATTRwt) while 11 patients showed left ventricular hypertrophy not related to amyloidosis. In all AL patients, biopsy site was abdominal fat pad. In TTR amyloidosis, positive biopsy was present in 20/53 (38\%) patients and the positive biopsy site was abdominal fat in 12 patients and myocardium in 8 .

Twenty-six patients (5 AL, 6 ATTRm, 4 ATTRwt and $11 \mathrm{LVH}$ ) were part of an already published multicentre analysis. ${ }^{11}$ Demographic and clinical characteristics of three groups are reassumed in Table 1. 
At $99 \mathrm{mTc}-\mathrm{HMDP}$ scintigraphy only one out of 12 AL patients showed a Perugini 1 heart retention while all patients with TTR cardiac amyloidosis demonstrated heart uptake, with 5 Perugini visual score 1, 20 Perugini 2 and 28 Perugini 3 (Table 1). No cardiac uptake was evidenced in patients with $\mathrm{LVH}$ not due to amyloid deposition. Considering five patients with grade 1 scan in TTR group, a Perugini grade 2-3 cardiac uptake on a 99mTc-HMDP scintigraphy showed a $91 \%$ sensitivity and a $100 \%$ specificity.

As reported in Table 1, all the semi-quantitative assessment methods showed significantly higher values in ATTR patients without significant differences between $\mathrm{AL}$ and LVH patients.

For each semi-quantitative method, ROC curve analysis was performed to identify the best cut-off value to achieve the greatest accuracy in ATTR identification. As reported in Table 2, H/WB indices $(\mathrm{H} / \mathrm{WBr}$ and $\mathrm{H} / \mathrm{WBp}$ ) reached a $100 \%$ accuracy in ATTR identification, irrespectively of the method used to trace the whole-body ROI, while H/CL showed a slightly worse performance due to a false-negative patient that showed a clear HMDP lung retention. On the other hand, all the parameters based on a ratio between heart and a bone structure $(\mathrm{H} / \mathrm{S} ; \mathrm{H} / \mathrm{P} ; \mathrm{H} / \mathrm{P}-$ bkg) showed a diagnostic accuracy below the expectation with more misclassified patients compared to $\mathrm{H} /$ WB and H/CL ratios. In fact, H/P-bkg and H/P misclassified 2 and 3 patients, respectively, while $\mathrm{H} / \mathrm{S}$ reached the worst performance misidentifying six falsenegative patients.

Results of a subgroup analysis of semi-quantitative method according to Perugini visual score grading in the whole population are reassumed in Table 3.

All semi-quantitative indices showed significantly different values between Perugini 0 and Perugini $\geq 1$, with the exception of $\mathrm{H} / \mathrm{S}$, that showed no significantly different values between Perugini 0 and Perugini 1.

Table 1. Clinical and biohumoral characteristics

\begin{tabular}{|c|c|c|c|c|}
\hline & LVH $(n=11)$ & $A L(n=12)$ & $\operatorname{TTR}(n=53)$ & $\boldsymbol{P}$ \\
\hline Age, years & $78 \pm 6$ & $72 \pm 13$ & $78 \pm 7$ & .07 \\
\hline Gender $M / F$ & $10 / 1$ & $8 / 4$ & $43 / 6$ & .204 \\
\hline NT-proBNP, ng/ml & $3352 \pm 2677$ & $6871 \pm 6405$ & $4773 \pm 4046$ & .241 \\
\hline IVS, mm & $14.7 \pm 2.9$ & $16.6 \pm 2.1$ & $17.3 \pm 2.9$ & .015 \\
\hline LVPW, mm & $13.3 \pm 1.5$ & $13.8 \pm 2.4$ & $16.1 \pm 2.7$ * * & .003 \\
\hline LVEDV, ml & $95 \pm 33$ & $97 \pm 30$ & $74 \pm 30$ & .384 \\
\hline LVESV, ml & $42 \pm 23$ & $52.5 \pm 26.1$ & $30.1 \pm 13.6$ & .138 \\
\hline $\mathrm{EF} \%$ & $55 \pm 9$ & $59 \pm 7$ & $48 \pm 12$ & .053 \\
\hline $\mathrm{E} / \mathrm{E}^{\prime}$ & $15 \pm 6$ & $16 \pm 8$ & $17 \pm 6$ & .610 \\
\hline TAPSE, mm & $19 \pm 4$ & $17 \pm 3$ & $16 \pm 4$ & .345 \\
\hline Perugini score & & & & .0001 \\
\hline Perugini 0 & 11 & 11 & 0 & \\
\hline Perugini 1 & - & 1 & 5 & \\
\hline Perugini 2 & - & - & 20 & \\
\hline Perugini 3 & - & - & 28 & \\
\hline H/WBp & $1.8 \pm 0.7$ & $2.2 \pm 0.5$ & $6.0 \pm 1.6^{*}$ & .0001 \\
\hline $\mathrm{H} / \mathrm{WBr}$ & $1.8 \pm 0.7$ & $2.2 \pm 0.5$ & $5.9 \pm 1.6^{*}$ & .0001 \\
\hline $\mathrm{H} / \mathrm{P}$ & $0.9 \pm 0.2$ & $0.85 \pm 0.1$ & $1.8 \pm 0.42^{*}$ & .0001 \\
\hline $\mathrm{H} / \mathrm{S}$ & $1.6 \pm 0.3$ & $1.5 \pm 0.3$ & $2.8 \pm 0.8^{*}$ & .0001 \\
\hline H/P-bkg & $0.4 \pm 0.3$ & $0.3 \pm 0.1$ & $0.9 \pm 0.4^{*}$ & .0001 \\
\hline $\mathrm{H} / \mathrm{CL}$ & $1.0 \pm 0.1$ & $1.1 \pm 0.1$ & $1.8 \pm 0.3^{*}$ & .0001 \\
\hline
\end{tabular}

$A L$, Light-chain amyloidosis; $E F$, ejection fraction; $H / C L$, Cardiac Uptake/Contralateral Lung Index; $H / P$-bkg, Heart/pelvis ratio with tissue background correction; $H / P$, Heart/Pelvis Index, $H / S$, Heart/Skull Index; $H / W B r$, Heart/whole-body rectangular ROI ratio; $H / W B p$, Heart/whole-body profile ROI ratio; IVS, interventricular septum; $L V E D V$, left ventricular end-diastolic volume; $L V E S V$, left ventricular end-systolic volume; $L V H$, left ventricular hypertrophy; PW, posterior wall; TAPSE, Tricuspid Annular Plane Systolic Excursion; TTR, transthyretin amyloidosis

Post hoc analysis ${ }^{*} P<.05$ TTR vs others

${ }^{*}{ }^{*}<.05$ TTR vs LVH 


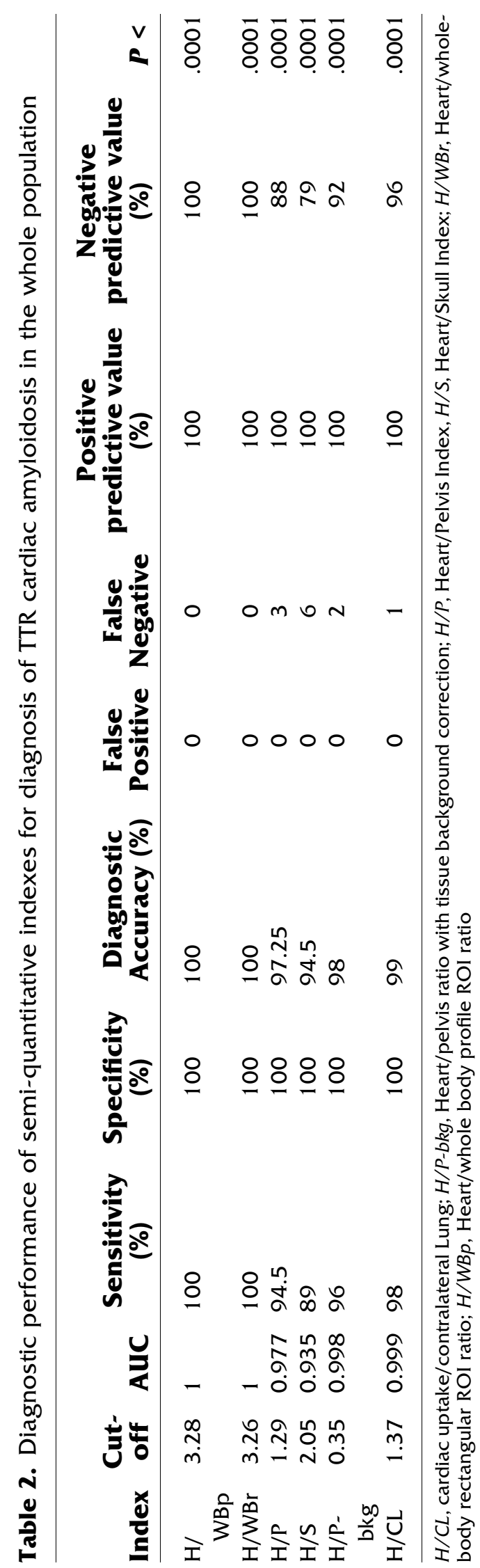


Table 3. Semi-quantitative index values by Perugini visual score grading

\begin{tabular}{lccccc}
\hline & Perugini $\mathbf{~ N}=\mathbf{2 2}$ & Perugini $\mathbf{~ N}=\mathbf{6}$ & Perugini $2 \boldsymbol{N}=\mathbf{2 0}$ & Perugini $3 \boldsymbol{N}=\mathbf{2 8}$ & $\boldsymbol{P}<$ \\
\hline $\mathrm{H} / \mathrm{WBp}$ & $1.9 \pm 0.6^{*}$ & $4.3 \pm 0.7^{\S}$ & $5.9 \pm 1.7$ & $6.4 \pm 1.5$ & .0001 \\
$\mathrm{H} / \mathrm{WBr}$ & $2.0 \pm 0.6^{*}$ & $4.3 \pm 0.6^{\S}$ & $5.8 \pm 1.8$ & $6.3 \pm 1.5$ & .0001 \\
$\mathrm{H} / \mathrm{P}$ & $0.87 \pm 0.15^{*}$ & $1.6 \pm 0.6$ & $1.8 \pm 0.41$ & $1.9 \pm 0.4$ & .0001 \\
$\mathrm{H} / \mathrm{S}$ & $1.6 \pm 0.3^{*}$ & $1.9 \pm 0.6$ & $2.7 \pm 0.7^{£}$ & $3.0 \pm 0.7^{£}$ & .0001 \\
$\mathrm{H} / \mathrm{P}-\mathrm{bkg}$ & $0.2 \pm 0.5^{*}$ & $0.4 \pm 0.2^{\S}$ & $0.8 \pm 0.4$ & $1.0 \pm 0.4$ & .0001 \\
$\mathrm{H} / \mathrm{CL}$ & $1.0 \pm 0.1^{*}$ & $1.5 \pm 0.2$ & $1.8 \pm 0.4$ & $1.9 \pm 0.3^{\AA}$ & .0001
\end{tabular}

$H / C L$, cardiac uptake/contralateral lung; $H / P$-bkg, Heart/pelvis ratio with tissue background correction; $H / P$, Heart/Pelvis Index, $H / S$, Heart/Skull Index; $H / W B r$, Heart/whole-body rectangular ROI ratio; $H / W B p$, Heart/whole-body profile ROI ratio

Post hoc analysis: ${ }^{*} P<.05$ for Perugini 0 vs others; ${ }^{\S} P<.05$ for Perugini 1 vs others; ${ }^{£} P<.05$ vs Perugini 0 and Perugini 1 ; $\& P<.05$ for Perugini 3 vs Perugini 0 and Perugini 1

H/WB indices showed significantly different values between Perugini 0 and Perugini 1 and, between Perugini 1 and Perugini 2-3, while H/CL showed a significant difference between Perugini 0 and Perugini 1 but no significant difference between Perugini 1 and Perugini 2. Surprisingly, no semi-quantitative method was able to show a significant difference between Perugini 2 and Perugini 3 subgroups. ROC curve analysis identified cut-off values able to discriminate subjects with Perugini 2-3 versus Perugini 0-1 (Table 4). All the methods reached an acceptable sensitivity and specificity, with $\mathrm{H} / \mathrm{P}-\mathrm{bkg}$ that reached the highest area under the curve (AUC) followed by $\mathrm{H} / \mathrm{WB}$ (both "rectangular" and "profile") while H/S and H/P had the lowest AUC.

Analysis of inter-observer and intra-observer variability is reported in Table 5. H/CL showed the greatest repeatability and reproducibility, despite interobserver analysis of data resulted in re-classification of one patient. H/WB indices, both rectangular and profile, showed comparable results, with adequate inter- and intra-observer variability. When compared to $\mathrm{H} / \mathrm{CL}$, they showed less consistency, but still resulted in higher robustness: no patient was reclassified after inter-observer analysis of data, without major consequences on diagnostic performance. Higher variability is somehow an expected finding, since $\mathrm{H} / \mathrm{WB}$ indices depend upon selection of multiple ROIs, while $\mathrm{H} / \mathrm{CL}$ relies on just two ROIs, thus reducing the place for measurement changes. Of note, $\mathrm{H} / \mathrm{S}$ had the highest variability both at inter- and intraobserver analysis; such a finding may result from a combination of both the number of ROIs (in anterior and posterior projection) required, and the difficult definition of skull ROI using a rectangular contour. Despite high repeatability, H/P-bkg was affected by lower inter-observer reproducibility of measurement.

\section{DISCUSSION}

To the best of our knowledge, this is the first research comparing several 99mTC-HMDP total body scintigraphy semi-quantitative indices in patients undergoing multidisciplinary evaluation for suspected cardiac amyloidosis.

Our data confirm that patients with TTR cardiac amyloidosis show 99mTc-HMDP heart retention with commonly a Perugini $2-3$ visual score, while AL amyloidosis less often shows heart retention and, at least in our population, presents with lower visual score. Moreover, our data show that all the semi-quantitative parameters are significantly increased in the TTR cardiac amyloid subgroup. On the other hand, different semi-quantitative heart retention indices showed different accuracy in cardiac TTR amyloid identification (Table 2). The ratio between heart retention and wholebody retention showed the best accuracy irrespectively of the method used to identify the whole-body ROI. This finding is consistent with our previous work, where a cut-off of 3.3 at H/WB showed a $100 \%$ accuracy in cardiac ATTR identification. ${ }^{11} \mathrm{H} / \mathrm{S}$ ratio showed unacceptable sensitivity with six patients with TTR cardiac amyloidosis misclassified as false negative, while the newly proposed heart to pelvis ratio, showed an intermediate accuracy with better results if corrected by background. H/CL demonstrated a good accuracy in cardiac TTR amyloid identification, with only one falsenegative result. Re-analysis of 99mTc-HMDP scintigraphy of this subject showed a clear lung retention that, as previously reported by our group, could have reduced the ratio between heart and lung resulting in a misidentification. ${ }^{17}$

H/S lowest sensitivity could be reasonably explained by two factors: the presence of increased skull uptake and/or a low heart uptake. In fact, skull and 
maxillofacial bones show highly variable retention of phosphonates; its enhancement, for instance in hyperostosis (a common paraphysiological condition) and sinusitis, increases the denominator of the ratio, reducing the value of the index. Second, among H/S falsenegative patients, we observed a prevalence of Perugini 1 patients, that showed lower value of heart uptake.

On the other hand, the better accuracy of H/P-bkg compared to $\mathrm{H} / \mathrm{P}$ could be explained by tissue background correction that minimizes the undesired effects of aspecific tracer distribution and background noise.

As recently suggested by Rapezzi and coauthors, ${ }^{18}$ while different bone tracers (99mTc-PYP, 99mTc-HMDP and $99 \mathrm{mTc}-\mathrm{DPD})$ seem to be interchangeable for the diagnosis of cardiac ATTR, bone tracers are not equally able to image non-cardiac TTR amyloidosis. Hutt and colleagues demonstrated that a consistent percentage of patients with TTR amyloidosis (70/77) show a clear 99mTc-DPD muscular retention, particularly in gluteal, shoulder region and in the abdominal wall. ${ }^{5}$ These data have been confirmed by a larger study by the same group in which authors demonstrated, in a cohort of TTR amyloidosis patients, that soft tissue to femur ratio (a reasonable marker of extracardiac amyloid infiltration) was significantly different between each Perugini grade of $99 \mathrm{mTc}-\mathrm{DPD}$ scan, progressively increasing from grade 1 to grade 3 visual score. ${ }^{19}$ On the other hand, Sperry and coauthors reported that no muscular retention could be evidenced in a cohort of 57 patients with TTR cardiac amyloidosis, in an advanced phase of the disease, who underwent $99 \mathrm{mTc}-\mathrm{PYP}$ scintigraphy. ${ }^{20}$ In our cohort, few patients (1 (5\%) AL patient; 5 (9\%) TTR patients, $0 \mathrm{LVH}$ patients) showed muscular uptake, suggesting that HMDP acts at an intermediate level between PYP and DPD regarding muscular tracer retention. Therefore, in our cohort the good accuracy reached by $\mathrm{H} / \mathrm{WBr}$ and $\mathrm{H} / \mathrm{WBp}$ in cardiac TTR amyloidosis identification could be also explained by the lack of diffuse muscular tracer retention. Conversely, the major technical concern about H/WB ratio is the overlap between heart and sternum, which could lead to an overestimation of heart retention. Otherwise the determinants of total counts measured in $\mathrm{H} / \mathrm{WB}$ indices (administered dose, time of acquisition and urinary radiotracer excretion) affects proportionally cardiac uptake and WB retention contributing to the robustness of these indices.

Heart to bones ratios achieved a worse accuracy in cardiac TTR amyloid identification especially in Perugini 1 patients, with false-negative result in one or more heart-to-bones indices in $80 \%$ of cases. This lack of accuracy could be explained by a combination of lower cardiac uptake and bone hyperactivity, which could result in lower values, and consequently in false-negative identification. 
Table 5. Intra-observer and inter-observer variability analysis

\begin{tabular}{|c|c|c|c|c|c|c|}
\hline \multirow[b]{2}{*}{ Index } & \multicolumn{3}{|c|}{ Intra-observer variability } & \multicolumn{3}{|c|}{ Inter-observer variability } \\
\hline & ICC & CI $95 \%$ & $\boldsymbol{P}$ & ICC & CI $95 \%$ & $\boldsymbol{P}$ \\
\hline $\mathrm{H} / \mathrm{WBp}$ & 0.93 & $0.740-976$ & $<.001$ & 0.94 & $0.796-0.977$ & $<.001$ \\
\hline $\mathrm{H} / \mathrm{WBr}$ & 0.92 & $0.630-973$ & $<.001$ & 0.93 & $0.825-0.975$ & $<.001$ \\
\hline $\mathrm{H} / \mathrm{P}$ & 0.95 & $0.871-0.979$ & $<.001$ & 0.94 & $0.869-0.978$ & $<.001$ \\
\hline $\mathrm{H} / \mathrm{S}$ & 0.82 & $0.545-0.930$ & $<.001$ & 0.77 & $0.249-0.922$ & $<.001$ \\
\hline H/P-bkg & 0.98 & $0.955-0.994$ & $<.001$ & 0.89 & $0.744-0.958$ & $<.001$ \\
\hline $\mathrm{H} / \mathrm{CL}$ & 0.98 & $0.963-0.944$ & $<.001$ & 0.99 & $0.967-0.995$ & $<.001$ \\
\hline
\end{tabular}

$H / C L$, cardiac uptake/contralateral lung; $H / P$-bkg, Heart/pelvis ratio with tissue background correction; $H / P$, Heart/Pelvis Index, $H / S$, Heart/Skull Index; $H / W B r$ Heart/whole-body rectangular ROI ratio; $H / W B p$, Heart/whole-body profile ROI ratio

As previously reported, semi-quantitative method could be a useful tool to correctly identify patients with TTR cardiomyopathy. Moreover, semi-quantitative index could be a help to correctly objectify heart retention and correctly classify the patient in the right Perugini grade.

Since the seminal work by Gillmore and coauthors, ${ }^{14}$ a correct identification of Perugini grade has reached a great clinical impact, especially in the hedge between Perugini 1 and Perugini 2. In fact, in the absence of a monoclonal gammopathy, a Perugini grade $\geq 2$ at scintigraphy allows a diagnosis of TTR cardiac amyloidosis, avoiding endomyocardial biopsy and its inherent risks. Our research demonstrated that, on a subgroup analysis according to Perugini visual score, semi-quantitative methods showed unexpected and heterogeneous results.

Surprisingly $\mathrm{H} / \mathrm{CL}$ does not show significantly different values between Perugini 1 and Perugini 2 patients. Moreover, no semi-quantitative index showed significantly different values between patients with Perugini 2 and Perugini 3 visual score. On ROC curve analysis $\mathrm{H} / \mathrm{WBr}$ and $\mathrm{H} / \mathrm{P}-\mathrm{bkg}$ showed an acceptable accuracy in discrimination between Perugini $0-1$ and Perugini 2-3. Unfortunately, as previously discussed, despite a good performance in Perugini 1 vs Perugini 23 discrimination, H/P-bkg shows a slightly lower accuracy in identifying cardiac TTR amyloidosis patients.

Therefore, our observations as a whole would suggest that $\mathrm{H} / \mathrm{WB}$ ratios (both profile and rectangular ROI) could be a reliable semi-quantitative method in the interpretation of 99mTC-HMDP scintigraphy, since they achieved the best performance in correctly identifying cardiac TTR amyloidosis, while at the same time showed a good diagnostic accuracy in correctly classifying Perugini 1 vs Perugini 2-3 subgroups, a discrimination with major clinical implication; furthermore $\mathrm{H} / \mathrm{WB}$ ratios allowed for a good repeatability and reproducibility, with the highest robustness of diagnostic performance. Due to the comparable results in HR/WB ratios, irrespectively to the method used to trace the whole-body ROI, rectangular WB ROI could be recommended as the easiest and time saving technique to evaluate WB tracer retention.

\section{NEW KNOWLEDGE GAINED}

In a cohort of patients that underwent $99 \mathrm{mTc}$ HMDP in a clinical suspicious of cardiac amyloidosis, H/WB (rectangular or profile) ratios are the best semiquantitative indices able to correctly identify patients with TTR cardiac amyloidosis. H/CL seems to be less accurate mainly in case of simultaneous heart and lung bone tracer retention.

In a real-world setting, H/WB could be the semiquantitative methods of choice to help clinicians to correctly identify TTR cardiac amyloidosis patients and differentiate between Perugini 1 and Perugini 2-3, a discrimination with major clinical implication.

\section{Disclosure}

The authors have no conflict of interest in this manuscript preparation.

\section{References}

1. Perfetto F, Cappelli F, Bergesio F, Ciuti G, Porciani MC, Padeletti L. Moggi pignone a cardiac amyloidosis: The heart of the matter. Intern Emerg Med 2013;8:191-203.

2. Perugini E, Guidalotti PL, Salvi F, Cooke RM, Pettinato C, Riva $\mathrm{L}$, et al. Noninvasive etiologic diagnosis of cardiac amyloidosis using $99 \mathrm{mTc}-3,3$-diphosphono-1,2-propanodicarboxylic acid scintigraphy. J Am Coll Cardiol 2005;46:1076-84. 
3. Rapezzi C, Quarta CC, Guidalotti PL, Pettinato C, Fanti S, Leone O, et al. Role of $(99 \mathrm{~m}) \mathrm{Tc}-\mathrm{DPD}$ scintigraphy in diagnosis and prognosis of hereditary transthyretin related cardiac amyloidosis. JACC Cardiovasc Imaging 2011;4:659-70.

4. Longhi S, Guidalotti PL, Quarta CC, Gagliardi C, Milandri A, Lorenzini M, Potena L, et al. Identification of TTR related subclinical amyloidosis with 99mTc-DPD scintigraphy. JACC Cardiovasc Imaging 2014;7:531-2.

5. Hutt DF, Quigley AM, Page J, Hall ML, Burniston M, Gopaul D, et al. Utility and limitations of 3,3-diphosphono-1,2-propanodicarboxylic acid scintigraphy in systemic amyloidosis. Eur Heart J Cardiovasc Imaging. 2014;15:1289-98.

6. Bokhari S, Castano A, Pozniakoff T, Deslisle S, Latif F, Maurer MS. (99m)Tc-pyrophosphate scintigraphy for differentiating lightchain cardiac amyloidosis from the transthyretin-related familial and senile cardiac amyloidoses. CircCardiovasc Imaging 2013;6:195-201.

7. Hongo M, Hirayama J, Fujii T, Kinoshita O, Tanaka M, Okubo S. Early identification of amyloid heart disease by technetium-99mpyrophosphate scintigraphy: a study with familial amyloid polyneuropathy. Am Heart J 1987;113:654-62.

8. Wizenberg TA, Muz J, Sohn YH, Samlowski W, Weissler AM. Value of positive myocardial technetium-99m-pyrophosphate scintigraphy in the noninvasive diagnosis of cardiac amyloidosis. Am Heart J 1982;103:468-73.

9. Glaudemans AW, van Rheenen RW, van den Berg MP, Noordzij $\mathrm{W}$, Koole $\mathrm{M}$, Blokzijl $\mathrm{H}$, et al. Bone scintigraphy with (99m)technetium-hydroxymethylene diphosphonate allows early diagnosis of cardiac involvement in patients with transthyretinderived systemic amyloidosis. Amyloid 2014;21:35-44.

10. Galat A, Rosso J, Guellich A, Van Der Gucht A, Rappeneau S, Bodez D, Guendouz S, et al. Usefulness of (99m)Tc-HMDP scintigraphy for the etiologic diagnosis and prognosis of cardiac amyloidosis. Amyloid 2015;22:210-20.

11. Cappelli F, Gallini C, Di Mario C, Costanzo EN, Vaggelli L, Tutino F, Ciaccio A, Bartolini S, Angelotti P, Frusconi S, Farsetti S, Vergaro G, Giorgetti A, Marzullo P, Genovesi D, Emdin M, Perfetto F. Accuracy of 99mTc-Hydroxymethylene diphosphonate scintigraphy for diagnosis of transthyretin cardiac amyloidosis. J Nucl Cardiol 2017.

12. Pradel S, Brun S, Victor G, Pascal P, Fournier P, Ribes D, LavieBadie Y, Galinier M, Carri ̃̃ D, Berry I, Lairez O; Toulouse Amyloidosis Research Network collaborators. Pattern of myocardial (99m)Tc-HMDP uptake and impact on myocardial function in patients with transthyretin cardiac amyloidosis. J Nucl Cardiol 2018. https://doi.org/10.1007/s12350-018-1316-6.

13. Harb SC, Haq M, Flood K, Guerrieri A, Passerell W, Jaber WA, Miller EJ. National patterns in imaging utilization for diagnosis of cardiac amyloidosis: A focus on Tc99m-pyrophosphate scintigraphy. J Nucl Cardiol. 2017;24:1094-7. https://doi.org/10.1007/ s12350-016-0478-3.

14. Gillmore JD, Maurer MS, Falk RH, Merlini G, Damy T, Dispenzieri A, et al. Nonbiopsy diagnosis of cardiac transthyretin amyloidosis. Circulation 2016;133:2404-12.

15. Gertz M, Merlini G. Definition of organ involvement and response to treatment in AL amyloidosis: an updated consensus opinion. Amyloid 2010;17:48-9.

16. R.M. Lang, L.P. Badano, V. Mor-Avi, J. Afilalo, A. Armstrong, L. Ernande, et al., Recommendations for cardiac chamber quantification by echocardiography in adults: an update from the American Society of Echocardiography and the European Association of Cardiovascular Imaging, J Am Soc Echocardiogr 2015;1:39.e14

17. Cappelli F, Gallini C, Costanzo EN, Tutino F, Ciaccio A, Vaggelli L, Bartolini S, Morini S, Martone R, Angelotti P, Frusconi S, Di Mario C, Perfetto F. Lung uptake during 99mTc-hydroxymethylene diphosphonate scintigraphy in patient with TTR cardiac amyloidosis: An underestimated phenomenon. Int J Cardiol 2018;1:346-50. https://doi.org/10.1016/j.ijcard.2017.10.027.

18. Rapezzi C, Gagliardi C, Milandri A. Analogies and disparities among scintigraphic bone tracers in the diagnosis of cardiac and non-cardiac ATTR amyloidosis. J Nucl Cardiol 2018. https://doi. org/10.1007/s12350-018-1235-6.

19. Hutt DF, Fontana M, Burniston M, Quigley AM, Petrie A, Ross JC, et al. Prognostic utility of the Perugini grading of 99mTc- DPD scintigraphy in transthyretin (ATTR) amyloidosis and its relationship with skeletal muscle and soft tissue amyloid. Eur Heart J Cardiovasc Imaging 2017;18:1344-50.

20. Sperry BW, Gonzalez MH, Brunken R, Cerqueira MD, Hanna M, Jaber WA. Non-cardiac uptake of technetium-99m pyrophosphate in transthyretin cardiac amyloidosis. J Nucl Cardiol 2018. https://d oi.org/10.1007/s12350-017-1166-7.

Publisher's Note Springer Nature remains neutral with regard to jurisdictional claims in published maps and institutional affiliations. 